Artículo científico

(Original paper)

\title{
EREMOBLATTA ATTICOLA SP. N. (CORYDIIDAE: CORYDIINAE), NUEVA CUCARACHA MIRMECÓFILA DE MÉXICO
}

\section{EREMOBLATTA ATTICOLA SP. N. (CORYDIIDAE: CORYDIINAE), NEW MYRMECOPHILE COCKROACH OF MEXICO}

\author{
JULio CeSAR ESTRADA-ÁLVAREZ ${ }^{1,2 *}$, PATRICIA ROJAS ${ }^{3}$ \\ ${ }^{1}$ Museo Universitario de Historia Natural Dr. Manuel M. Villada UAEMex, Inst. Literario 100, Colonia Centro, Toluca, Estado \\ México. C.P. 50000.<micraten@yahoo.com.mx> \\ ${ }^{2}$ Entomological Research, Bo. San Mateo, Metepec, Estado de México, México. C.P. 52140. <micraten@yahoo.com.mx> \\ ${ }^{3}$ Red de Biodiversidad y Sistemática. Laboratorio de Invertebrados del Suelo, Instituto de Ecología A.C. (INECOL). Ap. Postal \\ 63, 91000, Xalapa, Veracruz, México.<patricia.rojas@inecol.mx> \\ *Autor corresponsal: <micraten@yahoo.com.mx> \\ Recibido: 19/09/2019; aceptado: 26/04/2020; publicado en línea: 10/06/2020 \\ Editor responsable: Arturo Bonet Ceballos
}

Estrada-Álvarez, J. C., Rojas, P. (2020) Eremoblatta atticola sp. n. (Corydiidae: Corydiinae), nueva cucaracha mirmecófila de México. Acta Zoológica Mexicana (nueva serie), 36, 1-14. https://doi.org/10.21829/azm.2020.3612240

RESUMEN. Se describe una nueva especie del género Eremoblatta Rehn, 1903, con base en material colectado en detritos de Atta mexicana (Smith 1858), en Querétaro, México.

Palabras clave: Eremoblatta; Atta; tipo; detritos; México; mirmecófila

Estrada-Álvarez, J. C., Rojas, P. (2020) Eremoblatta atticola sp. n. (Corydiidae: Corydiinae), new myrmecophile cockroach of Mexico. Acta Zoológica Mexicana (nueva serie), 36, 1-14. https://doi.org/10.21829/azm.2020.3612240

ABSTRACT. A new species of the genus Eremoblatta Rehn, 1903 is described based on material collected in detritus from Atta mexicana (Smith 1858) in Querétaro, Mexico.

Key words: Eremoblatta; Atta; type; detritus; Mexico; myrmecophile

\section{INTRODUCCIÓN}

De las 4,641 especies de cucarachas (Blattodea s. lato) descritas a nivel mundial (Beccaloni \& Eggleton, 2011; 2013), solamente 24 son consideradas mirmecófilas, es decir, que están asociadas de alguna manera con una o varias especies de hormigas. Estas cucarachas mirmecófilas pertenecen a 12 géneros, cinco subfamilias y tres familias, la mayoría del continente americano (Cuadro 1). 
Cuadro 1. Cucarachas mirmecófilas y asociaciones.

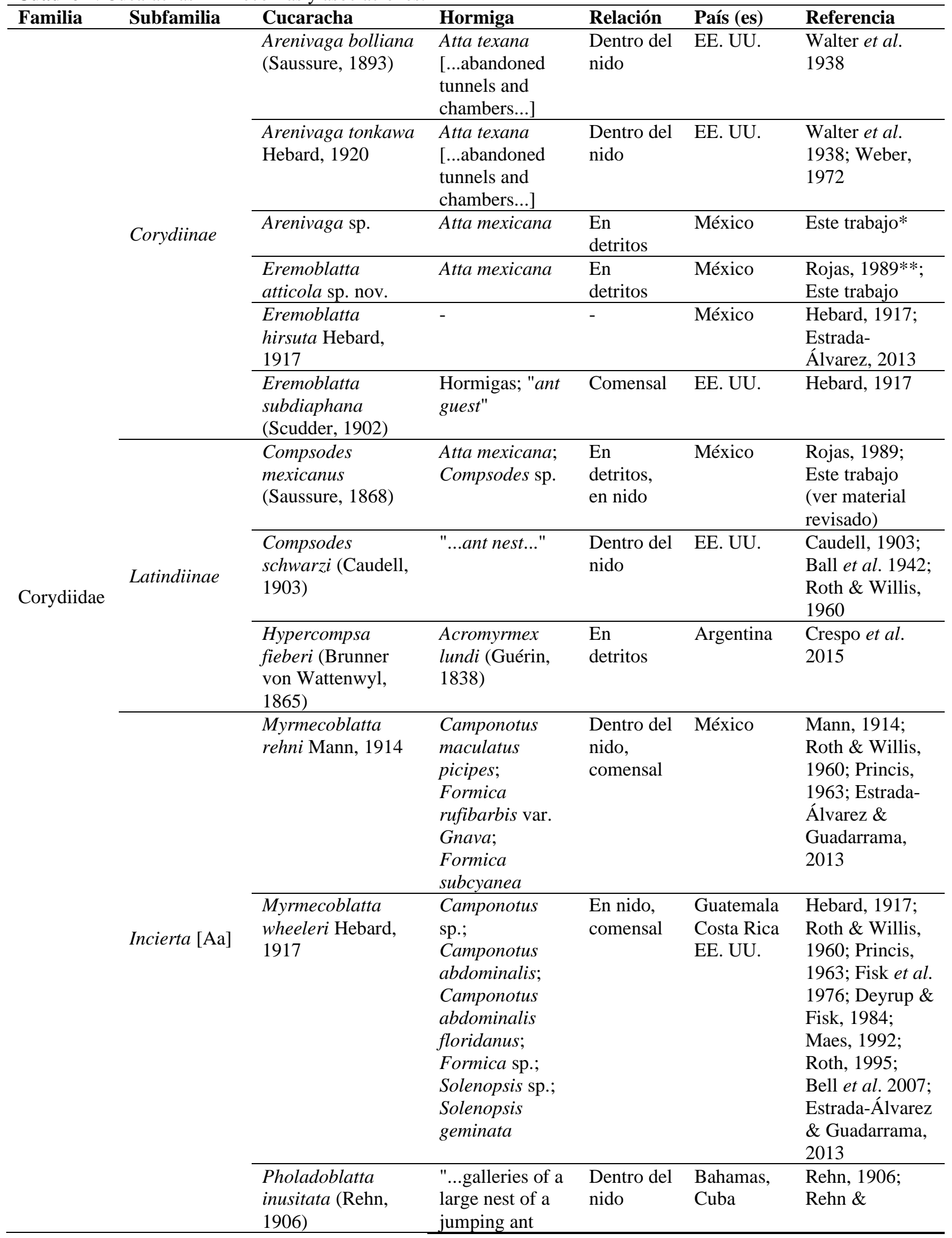




\begin{tabular}{|c|c|c|c|c|c|c|}
\hline Familia & Subfamilia & Cucaracha & Hormiga & Relación & País (es) & Referencia \\
\hline & & & $\begin{array}{l}\text { (Odontomachus } \\
\text { haematodes } \\
\text { insularis..." }\end{array}$ & & & $\begin{array}{l}\text { Hebard, 1927; } \\
\text { Princis, } 1963\end{array}$ \\
\hline \multirow{9}{*}{ Ectobiidae } & Nyctiborinae & $\begin{array}{l}\text { Nyctibora acaciana } \\
\text { Deans \& Roth, } \\
2003\end{array}$ & $\begin{array}{l}\text { Pseudomyrmex } \\
\text { sp. }\end{array}$ & $\begin{array}{l}\text { Pega las } \\
\text { ootecas } \\
\text { cerca de } \\
\text { los nidos } \\
\text { en acacias } \\
\end{array}$ & Costa Rica & $\begin{array}{l}\text { Deans \& Roth, } \\
\text { 2003; Bell et al. } \\
2007\end{array}$ \\
\hline & Blattellinae & $\begin{array}{l}\text { Pseudoanaplectinia } \\
\text { yumotoi Roth, } 1995\end{array}$ & $\begin{array}{l}\text { Crematogaster } \\
\text { sp.; } \\
\text { Crematogaster } \\
\text { deformis }\end{array}$ & Sin datos & Malasia & $\begin{array}{l}\text { Roth, 1995; } \\
\text { Bell et al. } 2007 .\end{array}$ \\
\hline & \multirow{7}{*}{$\begin{array}{l}\text { Blattellinae } \\
\text { [Aa] }\end{array}$} & Attaphila sp. 1 & $\begin{array}{l}\text { Atta colombica; } \\
\text { Acromyrmex } \\
\text { octospinosu }\end{array}$ & $\begin{array}{l}\text { En la } \\
\text { masa de } \\
\text { hongos }\end{array}$ & Panamá & $\begin{array}{l}\text { Nehring et al. } \\
2016\end{array}$ \\
\hline & & Attaphila sp. 2 & Atta cephalotes & Comensal & Guyana & $\begin{array}{l}\text { Beebe, 1921; } \\
\text { Roth \& Willis, } \\
1960\end{array}$ \\
\hline & & $\begin{array}{l}\text { Attaphila aptera } \\
\text { Bolívar, } 1905\end{array}$ & $\begin{array}{l}\text { Acromyrmex } \\
\text { octospinosus } \\
\text { [=Atta } 8- \\
\text { spinosa } \text { Reiche } \\
\text { (det. Forel)]; } \\
\text { Atta } \text { sp. pos. } \\
\text { Atta cephalotes }\end{array}$ & $\begin{array}{l}\text { En la } \\
\text { masa de } \\
\text { hongos }\end{array}$ & $\begin{array}{l}\text { Colombia } \\
\text { Surinam }\end{array}$ & $\begin{array}{l}\text { Bolivar, 1905; } \\
\text { Bruijning, } \\
\text { 1959; Roth \& } \\
\text { Willis, 1960; } \\
\text { Princis, 1963 }\end{array}$ \\
\hline & & $\begin{array}{l}\text { Attaphila sexdentis } \\
\text { Bolívar, } 1905\end{array}$ & $\begin{array}{l}\text { Atta sexdens } \\
\text { [=Atta sexdens } \\
\text { en Bolívar, } \\
\text { 1905: 137] }\end{array}$ & $\begin{array}{l}\text { Dentro del } \\
\text { nido, } \\
\text { comensal }\end{array}$ & Brasil & $\begin{array}{l}\text { Bolívar, 1905; } \\
\text { Roth \& Willis, } \\
\text { 1960; Princis, } \\
1963\end{array}$ \\
\hline & & $\begin{array}{l}\text { Attaphila schuppi } \\
\text { Bolívar, } 1905\end{array}$ & $\begin{array}{l}\text { Acromyrmex } \\
\text { niger [=Atta } \\
\text { nigra en } \\
\text { Bolívar, 1905: } \\
\text { 138] }\end{array}$ & Comensal & Brasil & $\begin{array}{l}\text { Bolívar, 1905; } \\
\text { Roth \& Willis, } \\
\text { 1960; Princis, } \\
1963\end{array}$ \\
\hline & & $\begin{array}{l}\text { Attaphila fungicola } \\
\text { Wheeler, } 1900\end{array}$ & $\begin{array}{l}\text { Atta sp.; Atta } \\
\text { fervens; Atta } \\
\text { cephalotes; Atta } \\
\text { texana }\end{array}$ & $\begin{array}{l}\text { En la } \\
\text { masa de } \\
\text { hongos; } \\
\text { comensal } \\
\text { y forética }\end{array}$ & $\begin{array}{l}\text { EE. UU. } \\
\text { Guyana, } \\
\text { Panamá? } \\
\text { Colombia }\end{array}$ & $\begin{array}{l}\text { Wheeler, 1900, } \\
\text { 1907, 1910, } \\
\text { 1928; Roth \& } \\
\text { Willis, 1960; } \\
\text { Princis, 1963; } \\
\text { Roth, 1995; } \\
\text { Moser, 1964; } \\
\text { Brossut, 1976; } \\
\text { Bell et al. 2007; } \\
\text { Rodríguez et al. } \\
\text { 2013; Phillips et } \\
\text { al. 2017 }\end{array}$ \\
\hline & & $\begin{array}{l}\text { Attaphila bergi } \\
\text { Bolívar, } 1901\end{array}$ & $\begin{array}{l}\text { Acromyrmex } \\
\text { lundi; } \\
\text { Acromyrmex } \\
\text { lobicornis; } \\
\text { Acromyrmex } \\
\text { silvestrii }\end{array}$ & $\begin{array}{l}\text { Dentro del } \\
\text { nido, } \\
\text { comensal }\end{array}$ & $\begin{array}{l}\text { Argentina } \\
\text { Uruguay }\end{array}$ & $\begin{array}{l}\text { Bolívar, 1901; } \\
\text { Wheeler, 1907; } \\
\text { Bruch, 1916; } \\
\text { Roth \& Willis, } \\
\text { 1960; Princis, } \\
\text { 1963; Roth, } \\
\text { 1995 }\end{array}$ \\
\hline
\end{tabular}




\begin{tabular}{|c|c|c|c|c|c|c|}
\hline Familia & Subfamilia & Cucaracha & Hormiga & Relación & País (es) & Referencia \\
\hline & & $\begin{array}{l}\text { Attaphila flava } \\
\text { Gurney, } 1937\end{array}$ & $\begin{array}{l}\text { Hormiga no } \\
\text { determinada }\end{array}$ & Comensal & Belice & $\begin{array}{l}\text { Gurney, 1937; } \\
\text { Roth \& Willis, } \\
\text { 1960; Princis, } \\
\text { 1963; Bell et al. } \\
2007\end{array}$ \\
\hline & \multirow{4}{*}{ Incierta [Ai] } & $\begin{array}{l}\text { Myrmeblattina } \\
\text { longipes (Chopard, } \\
\text { 1924) }\end{array}$ & $\begin{array}{l}\text { "Blattid for nest } \\
\text { of ant" [etiqueta } \\
\text { Holotipo]; } \\
\text { Eciton sp. } \\
\text { (sic)[=Odontom } \\
\text { achus affinis]; } \\
\text { Odontomachus } \\
\text { sp. }\end{array}$ & Comensal & Brasil & $\begin{array}{l}\text { Chopard, } \\
\text { 1924a,b; } \\
\text { Hancock, 1926; } \\
\text { Princis, 1963; } \\
\text { Beccaloni, 2014 }\end{array}$ \\
\hline & & $\begin{array}{l}\text { Phorticolea } \\
\text { boliviae Caudell, } \\
1923\end{array}$ & $\begin{array}{l}\text { Crematogaster } \\
\text { femoratus; } \\
\text { Crematogaster } \\
\text { limata }\end{array}$ & Comensal & Bolivia & $\begin{array}{l}\text { Caudell, 1923; } \\
\text { Roth \& Willis, } \\
\text { 1960; Princis, } \\
1963\end{array}$ \\
\hline & & $\begin{array}{l}\text { Phorticolea } \\
\text { testacea Bolívar, } \\
1905\end{array}$ & $\begin{array}{l}\text { Camponotus } \\
\text { rufipes }\end{array}$ & Comensal & Brasil & $\begin{array}{l}\text { Bolivar, 1905; } \\
\text { Roth \& Willis, } \\
\text { 1960; Princis, } \\
1963\end{array}$ \\
\hline & & $\begin{array}{l}\text { Atticola mortoni } \\
\text { Bolivar, } 1905\end{array}$ & $\begin{array}{l}\text { Camponotus } \\
\text { rufipes }\end{array}$ & Comensal & Brasil & $\begin{array}{l}\text { Bolivar, 1905; } \\
\text { Roth \& Willis, } \\
\text { 1960; Princis, } \\
1963\end{array}$ \\
\hline Blaberidae & Panchlorinae & $\begin{array}{l}\text { Pelloblatta reyesi } \\
\text { Gurney \& Roth, } \\
1972\end{array}$ & $\begin{array}{l}\text {...from chamber } \\
\text { of waste...of ant } \\
\text { nest (Atta sp.). } \\
\text { Atta cephalotes } \\
\text { [ID enmendada] }\end{array}$ & $\begin{array}{l}\text { En } \\
\text { detritos }\end{array}$ & México & $\begin{array}{l}\text { Gurney \& Roth, } \\
1972\end{array}$ \\
\hline
\end{tabular}

[Aa] = ex Familia Attaphilidae. [Ai] $]=$ ex familia Atticolidae. ${ }^{*}=$ ID errada $1 q$ como Eremoblatta sp. n. ca. hirsuta, en Rojas, 1989. **Eremoblatta sp. n. ca. hirsuta.

Las hormigas han sido consideradas "ingenieros del ecosistema" porque son capaces de moldear la presencia y abundancia de otras especies a través de la creación de nuevos hábitats (Jones et al., 1994). Atta mexicana (Smith) cultiva en el interior de sus nidos a un hongo simbiótico, alimento primordial de sus larvas; los desperdicios generados, son depositados en el exterior de sus nidos formando grandes montículos formados por material vegetal aprovechada por el hongo y otros residuos de la colonia, llamados detritos. Los detritos son ambientes ricos en materia orgánica y con un gradiente de humedad y temperatura constante a lo largo del año, por lo que resultan atractivos para gran diversidad de insectos durante parte o la totalidad de su ciclo de vida (Hendrichs \& Reyes-Castillo, 1963; Rojas, 1989). Del total de cucarachas viven en asociación directa con hormigas del género Atta Fabricius, 1805, únicamente cinco se han encontrado en el hábitat específico de las acumulaciones de detritos (Cuadro 1).

Rojas en 1989 dentro de su trabajo "Entomofauna asociada a los detritos de Atta mexicana (Smith) (Hymenoptera: Formicidae) en una zona árida del centro de México", reporta a una nueva especie del género Eremoblatta, quedando solamente como morfoespecies y referida así en Estrada-Álvarez (2013). Con la revisión de este material se describe esta nueva especie mirmecófila para México. El género Eremoblatta Rehn, 1903, es propuesto como subgénero de Homoeogamia Burmeister, 1838 por Rehn (1903) para contener a Homoeogamia subdiaphana Scudder, 1902 de EE. UU.; siendo Caudell en 1913, quien eleva el estatus a nivel de género. Hebard en 1917 describe la segunda especie Eremoblatta hirsuta Hebard, 1917, 
de México. En la actualidad Eremoblatta contiene a solamente dos especies con una distribución netamente neartica, en zonas áridas de México y EE. UU. (Beccaloni, 2014; Princis, 1963).

\title{
MATERIALES Y MÉTODOS
}

El material tipo fue revisado embebido en alcohol al $70 \%$. Las patas derechas I-III del macho holotipo y hembra paratipo fueron disectadas, para una mejor revisión. El abdomen del holotipo se disectó y puesto a digestión en ácido láctico $(90 \%)$ a una temperatura de $50{ }^{\circ} \mathrm{C}$, aproximadamente por tres horas, para retirar tejido blando. El material disectado fue preservado en un microvial con glicerina, etiquetado, almacenado en viales de vidrio junto al material al que correspondía en alcohol al $80 \%$ debidamente etiquetado. La terminología de la genitalia masculina sigue a Grandcolas (1994) y a Roth (2003). Las medidas se expresan en milímetros (mm). El mapa se realizó usando la capa de mapa hipsográfico de INEGI (INEGI, 2020).

\section{Colecciones.}

ANSP $=$ Academy of Natural Sciences of Philadelphia, Philadelphia, USA. $\mathrm{CER}=$ Colección entomológica, Entomological Research, Metepec, Estado de México, México. CNIN= Colección Nacional de Insectos, Instituto de Biología UNAM, CDMX, México. CBS= Departamento Biología de Suelos del INECOL, Xalapa, Veracruz, México. IEXA= Colección Entomológica INECOL, Sede Xalapa, Xalapa, Veracruz, México.

LESM= Laboratorio de Ecología y Sistemática de Microartrópodos, Facultad de Ciencias, UNAM, Ciudad de México, México.

\section{RESULTADOS}

\author{
BLATTODEA Brunner von Wattenwyl, 1882 \\ Corydioidea Saussure, 1864 \\ Corydiidae Saussure, 1864 \\ Corydiinae Saussure, 1864 \\ Eremoblatta Rehn, 1903 \\ Eremoblatta atticola sp. n. (Figs. 1-22)
}

Eremoblatta sp. n. ca. hirsuta Rojas, 1989: 9, 23, 34 [det. F. W. Fisk, 1983].

Eremoblata sp. Estrada-Álvarez, 2013: 272.

Material Tipo: $1 \widehat{\jmath}$ Holotipo; $1 \hat{\jmath}$ y $q$ Paratipo México, Querétaro, Km. 75.5 Tolimán-Higuerillas; 29/VIII/1982; P. Rojas col. (IEXA). $10^{\lambda}$ y 2 + Paratipo; Querétaro, Km. 75.5 Tolimán-Higuerillas; 27/I/1980; P. Rojas col. (CER). 1 đ Paratipo México, Querétaro, Cadereyta; 14/XI/1991; H. Braylovsky y E. Barrera cols. (CNIN).

Etimología: El epíteto especifico hacer referencia al género Atta Fabricius, 1805 (Formicidae), en cuyos detritos fue colectada la serie tipo.

Diagnosis: Eremoblatta atticola sp. n. está más relacionada con Eremoblatta hirsuta Hebard, 1917, se segrega de esta por la diferente nerviación de las tegminas y alas y la morfología de la lámina sub-genital (Figs. 7-14).

Descripción del macho (Holotipo) (Figs. 1-14). 11 largo total; 7 largo corporal y 4 ancho máximo. Coloración (Fig. 1): pronoto negro con dos maculas blancas mediales, en el margen anterior; meso y metasoma negros; abdomen de color chocolate; patas predominantemente negras, con las coxas 
notoriamente más claras, espinación de color chocolate; rostro negro, a excepción de los ocelos notoriamente blanquizcos; tegminas y alas de color humo, pronoto y patas con sedas piliformes de color dorado. Pronoto: 2 largo, 3.5 ancho y 2 alto, elevado dorso-ventralmente, cubriendo por completo al rostro, margen anterior profusamente procurvo, margen posterior ligeramente recurvo. Rostro: 1.4 largo, 1.3 ancho, ojos reniformes, distancia interocular de 0.6 ; ocelos 0.1 , blanquizcos con distancia interocelar de 0.5 , casi al borde de la frente; inserciones antenales 0.3, distancia entre inserciones de 0.4 (Figs. 1,2). Tegmina: textura coriácea, rebasando el borde posterior del abdomen, nerviación en figura 7. Ala: textura membranosa, nerviación en figura 8. Patas: fémur I con el margen antero-ventral sin espinas, suplidas por pilosidad larga, sin espinas apicales (Fig. 6) (espinación tipo $\mathrm{D}_{0}$, sensu Roth, 2003); tibia ensanchada apicalmente, con seis espinas, cinco en el margen apical y una espina ventro-apical, pilosidad larga en el margen retrolateral (Fig. 6); fémur II similar al anterior (Fig. 5); tibia ligeramente ensanchada apicalmente, con 15 espinas, cinco apicales, 10 en el margen retrolateral (cinco ventrales y cinco dorsales), pilosidad larga en la base del margen retrolateral (Fig. 5); fémur III similar a los anteriores (Fig. 4); tibia III con 18 espinas, dos prolaterales, tres apicales, 13 retrolaterales (nueve ventrales y cuatro dorsales) (Fig. 4). Tarsomeros I-IV de las patas I-III sin pulvillus, unas simétricas y simples sin arolium entre ellas. Abdomen: dorsalmente sin terguitos modificados, ventralmente con segmento IX con un conjunto de seis espínulas en la esquina antero-ventral (Fig. 11). Lamina supra-anal: más ancha que larga, dorsalmente con oquedad central y dos protuberancias centrales con cedas piliformes dirigidas dorso-anteriormente (Fig. 9); ventralmente con paraproctos subiguales, paraprocto derecho laminar, paraprocto izquierdo con ápice bífido. Cercos de unisegmentados con pseudosegmentación y tricolitos tergales conspicuos (Fig. 10). Lamina sub-genital (Hypandrium): dorsalmente con prolongación laminar en el lado derecho; ventralmente con cumulo de espínulas en el margen lateral (Figs. 11-13). Genitalia escleritos según figuras 14a, 14b: L3d y L2d ahusados y muy juntos, haciendo parecer a L3d bífido (según Hebard, 1917; Hopkins, 2014).

Descripción de la hembra (Paratipo) (Figs. 15-22). 10 largo, 7 ancho. Coloración: Dorsalmente chocolate, ventralmente más clara; con pilosidad de color dorado. Pronoto: 3 largo, 5 ancho; bordes lateroposteriores proyectados posteriormente. Mesonoto: 1.5 largo, 7 ancho; bordes latero-posteriores proyectados posteriormente. Metanoto: 1.5 largo, 7 ancho; bordes latero-posteriores proyectados posteriormente, cubriendo los bordes laterales de los segmentos abdominales I y II. Rostro: 1.7 largo 2.1 ancho; distancia entre inserciones antenales 1 ; ojos informes muy reducidos ( 0.5 largo máximo, 0.1 ancho máximo), distancia interocular de 1.4; ocelos ausentes (Fig. 17). Tegmina y alas ausentes. Patas: fémur I con el margen antero-ventral con 12 espínulas, pilosidad larga, sin espinas apicales (espinación similar al tipo $\mathrm{C}_{0}$, sensu Roth, 2003); tibia ensanchada apicalmente, con seis espinas, cuatro en el margen apical y una espina ventro-apical, pilosidad larga en el margen retrolateral (Fig. 18); fémur II con el margen anteroventral con siete espínulas y pilosidad larga; tibia ligeramente ensanchada apicalmente, con 11 espinas, cuatro apicales y siete en el margen retrolateral (cuatro ventrales y tres dorsales), pilosidad larga en la base del margen retrolateral (Fig. 19); fémur III con el margen antero-ventral con una espínula y pilosidad larga; tibia III con 18 espinas, dos prolaterales, siete apicales y nueve retrolaterales (siete ventrales y dos dorsales) (Fig. 20). Tarsomeros I-IV de las patas I-III sin pulvillus, uñas simétricas y simples sin arolium entre ellas. Coxas II y III con hilera de espínulas en el margen antero-ventral. Abdomen: dorsalmente segmentos I, II con los bordes laterales cubiertos por el metanoto; segmento IX y X (lamina supra-anal), solo visibles en vista posterior (Figs. 15, 16, 21); segmentos ventrales V y VI con hilera de espínulas. Lamina supra-anal: visible solamente en vista posterior, con el margen posterior medialmente emarginado; cercos unisegmentados (Fig. 21). Lamina sub-genital: borde posterior redondeado y dos hendiduras, donde reposan los cercos (Fig. 22). 


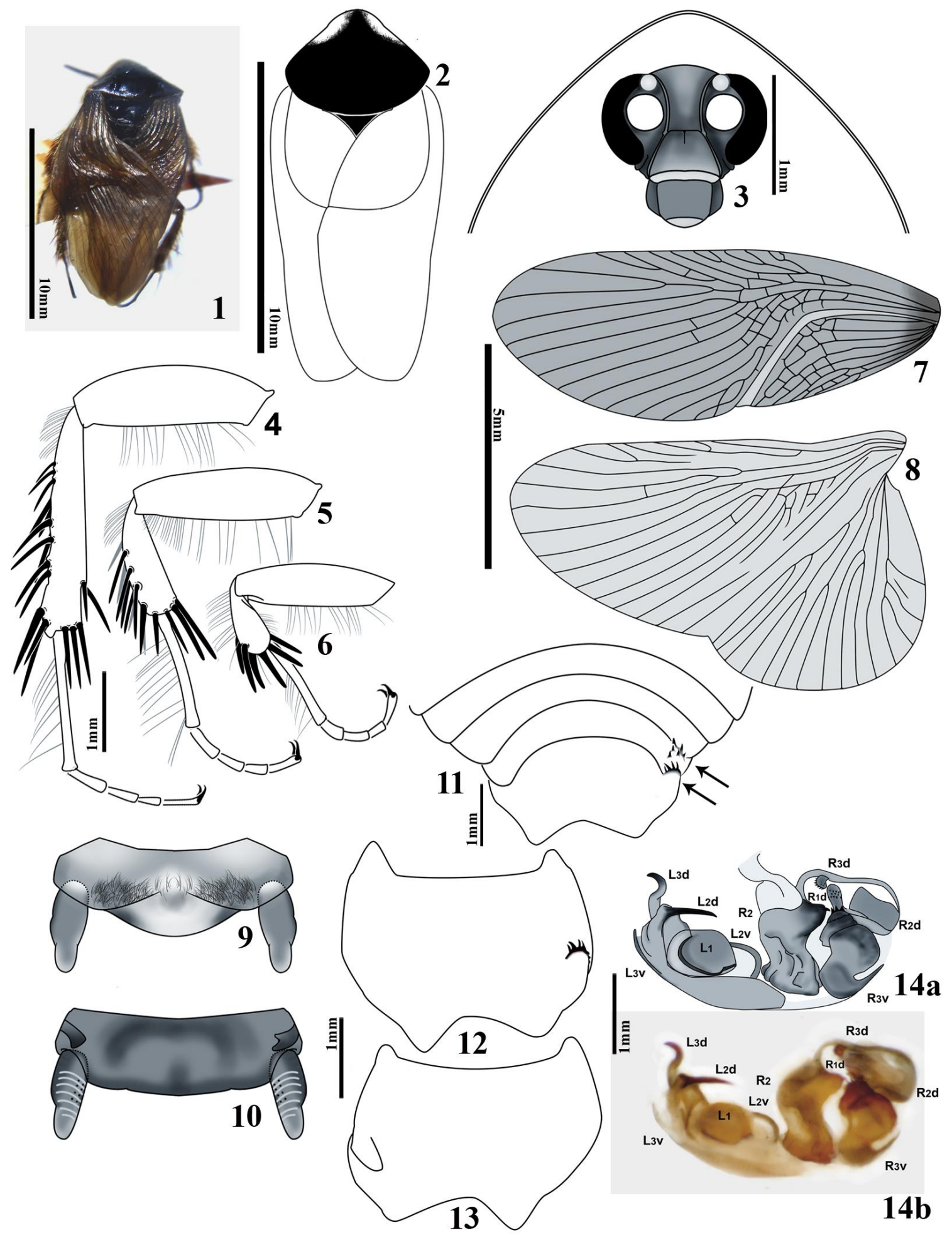

Figuras 1-14. Eremoblatta atticola sp. n. Holotipo (IEXA). 1,2) Vista dorsal; 3) Rostro; 4) Pata III; 5) Pata II; 6) Pata I; 7) Tegmina; 8) Ala; 9,10) Lamina supra-anal (9) Vista dorsal, (10) Vista ventral; 11) Segmentos ventrales VI$X$, mostrando cumulo de espínulas en el margen lateral de IX y X; 12,13) Lamina sub-genital (12), Vista ventral (13), Vista dorsal; 14) Genitalia en vista posterior, (14a) imagen digital, (14b) esquema (nomenclatura según Grandcolas, 1994). 


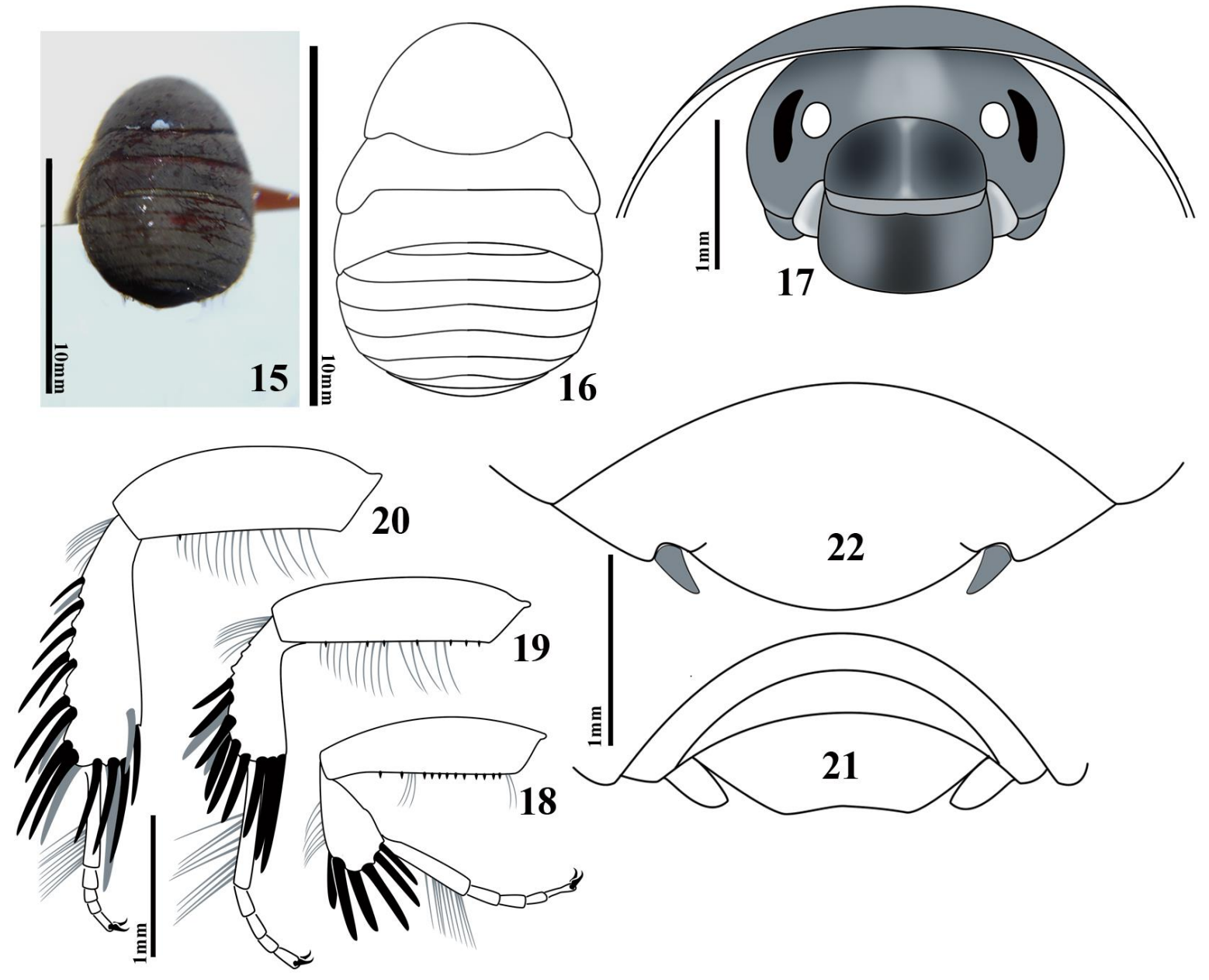

Fig. 15-22. Eremoblatta atticola sp. n. Paratipo (IEXA). 15,16) Vista dorsal; 17) Rostro; 18) Pata I; 19) Pata II; 20) Pata III; 21) Lamina supra-anal, en vista posterior; 22) Lamina sub-genital.

Distribución: Conocida para la localidad tipo México, Edo. Querétaro, Mpio. Cadereyta de Montes, Loc. Km. 75.5 Carretera 100, tramo San Pablo Tolimán-Higuerillas $\left(99^{\circ} 49^{\prime} 11.99^{\prime \prime} \mathrm{O}, 2^{\circ} 54^{\prime} 47.66 " \mathrm{~N}, 1,751\right.$

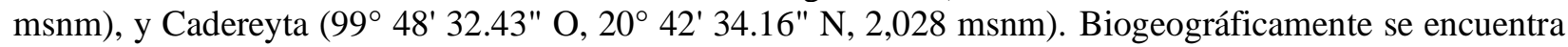
en la provincia del Altiplano Mexicano, en la región Neartica (Morrone, 2001; Morrone \& Márquez, 2008; Fig. 23).

Hábitat: La localidad tipo cuenta con clima seco a árido, templado; precipitación media anual de 377 mm, lluvias en verano y escasas en invierno (época de lluvias en mayo-octubre, secas noviembre-abril), temperatura media anual de $20.2^{\circ} \mathrm{C}$ y variación térmica de $7.7^{\circ} \mathrm{C}$, (BSohw" $(w)$ ). Vegetación dominante es matorral xerófilo (Rojas, 1989).

Bionomía: Los ejemplares fueron colectados en detritos externos de Atta mexicana (Smith 1858) (Rojas, 1989). $1 \overbrace{}^{\Uparrow}$ Holotipo y $q$ Paratipo colectados en plena cúpula.

Variación: Talla de los machos $11 \mathrm{~mm}$ largo total; largo corporal $7(\mathrm{~N}=2)$; Hembras $9(\mathrm{~N}=3)$. 


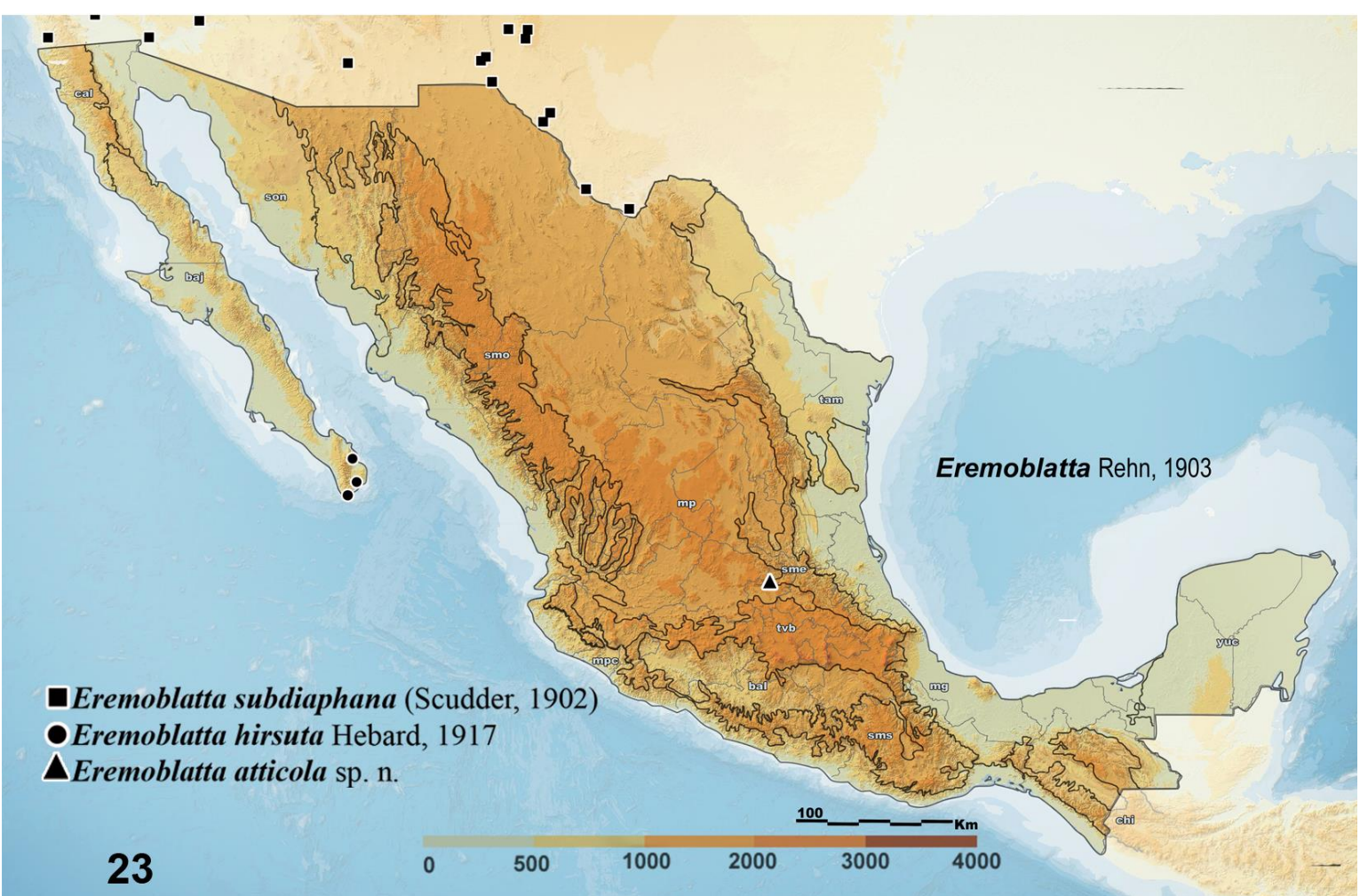

Figura 23. Distribución del género Eremoblatta Rehn, 1903 en México y áreas limítrofes. Provincias biogeográficas según Morrone (2001) y Morrone y Márquez (2008). Bal= Depresión del Balsas; $\boldsymbol{b} \boldsymbol{a j}=$ Baja California; $\boldsymbol{c a l}=$

California; $\boldsymbol{c h i}=$ Chiapas; $\boldsymbol{m g}=$ Golfo de México; $\boldsymbol{s} \boldsymbol{m e}=$ Sierra Madre Oriental; $\boldsymbol{s m o}=$ Sierra Madre Occidental; $\boldsymbol{s m} \boldsymbol{s}=$ Sierra Madre del Sur; $\boldsymbol{m p}=$ Altiplano mexicano; $\boldsymbol{m p} \boldsymbol{c}=$ Costas del pacífico mexicano; $\boldsymbol{s o n}=$ Sonora; $\boldsymbol{t a m}=$ Tamaulipas; $\boldsymbol{t} \boldsymbol{v} \boldsymbol{b}=$ Faja Volcánica Transmexicana; $\boldsymbol{y} \boldsymbol{u c}=$ Yucatán.

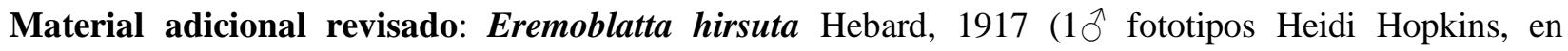
Beccaloni, 2014). Sierra El Tasti (Tosti(sic) en Hebard, 1917) [correctamente = Cerro El Taste], Lower

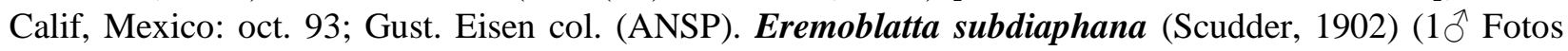
Topotipo Heidi Hopkins, en Beccaloni, 2014). Arenivaga aquila Hopkins, 20141 q Guerrero, a $3.8 \mathrm{~km}$ al oeste de Santa Cruz. Trampa de cebo, copro humano; 27 octubre 2005; Vaz de Melo, F. col (IEXA). Arenivaga dnopheros Hopkins, 20141 q Puebla, a $5 \mathrm{~km}$ Norte de Totoltepec. Trampa de cebo, copro humano; 26 diciembre 2005; Vaz de Melo, F. (IEXA). Arenivaga sp. (pos. A. florilega Hopkins, 2014) [det. Eremoblatta sp. n. hirsuta Hebard; det. F. W. Fisk 1983; determinación errónea] 2 우 México, km. 75.5 carr. Tolimán-Higuerillas, Qro. (III-C), en detritos de Atta mexicana, matorral de Larrea, $1540 \mathrm{~m}$ snm; 29/VIII/1982; P. Rojas col. (CBS). Homoeogamia mexicana Burmeister, 1838 (34: 25 $\partial^{\lambda}, 9+9+$ ). $1 \delta^{\lambda}$ Ciudad de México, C.U.; 14/XI/2017; G. Salgado col. (CNIN). 1ð̋ México, Hidalgo, Santiago de Ayala, Sección Norte; 24/VI/2006; C. Mayorga col. (CNIN). 3 đ̂ México, D.F., Instituto de Biología, UNAM; 16/V/2006; C. Mayorga col. (CNIN). 10ત México, D.F., Instituto de Biología, UNAM; 09/VI/2004; E. Mejorada col. (CNIN). 1ठ México, DF, C. U.; 14/VIII/2001; C. Mayorga col. (CNIN). 1ठ Uruapan, Michoacán; sin datos (CNIN). $1 \delta^{\Uparrow}$ El Sol, Tamanzuchale, S. L. P.; 05/V/1950; col. anónimo (CNIN). $1 \overbrace{}^{\lambda} \sin$

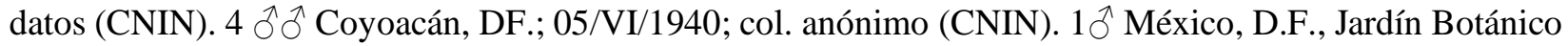
ext. C. U.; 07VI/1984; L. Vázquez col. (CNIN). 1 đ Catemaco, Veracruz, México; 15/VI/1972; E. Mariño col. (CNIN). 1ð̋ S. [San] Vicente, Gro.; [10-960-2?]; ex. Colección Bolívar (CNIN). 1ð Tacubaya; sin datos (CNIN). $1 \overbrace{}^{\lambda}$ Orizaba; sin datos (CNIN). $1{ }^{\lambda}$ Uruapan, Michoacán; VI/1942; col. anónimo (CNIN). $1{ }^{\lambda}$ México, Hidalgo, Mpio. Santiago de Ayala, Centro, sección norte (20²3’04``N, 9857'53”O; 04/X/2014; C. Mayorga col. (CNIN). 1ठ̂̉ México, Edo. de México, Sierra de Guadalupe; VIII-XII/2017; anónimo 
(CNIN). $1 \hat{\jmath} \sin$ datos (LESM). $2 \hat{\jmath} \widehat{\partial}, 3 q q$ México, Veracruz, Xalapa, santuario del bosque mesofilo de niebla; noviembre 2006; C. Sormani col. (IEXA). 1 + Hidalgo, Km. 18, Carr. Donguiño, 2270 m snm; 04/VI/1999; E. Barrera \& H. Braylovsky cols. (CNIN). 1 q México, DF.; 05/IX/2000; A. Castillo col. (CNIN). 1 q Actopan, Hidalgo; año 1936; C. C. Hoffmann col. (CNIN). 1 q Zempoala, Hgo.; VII/1940; D. Zavaleta col. (CNIN). 19 Alambaro(sic) [=Acámbaro]; sin datos (CNIN). 1 + Uruapan, Michoacán; VII/0000; Romano col. (CNIN). 1 q La Granja, Chis.; 13/VII/1967; anónimo (CNIN). Compsodes

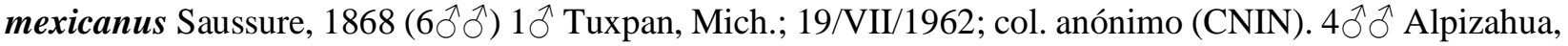

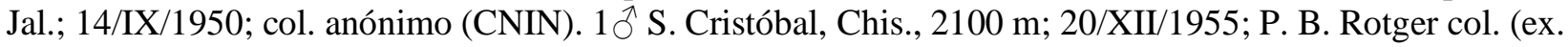

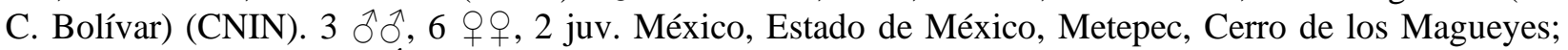
18/IX/2018; J. C. Estrada-Álvarez \& Balam Estrada F. cols. (CER). 1 đ̊ Camino rural entre Xalapa Enríquez y El Castillo cerca de La Laguna de Casa Blanca. Cafetal-Cañaveral. En un nido de hormigas Camponotus, ba jo piedra de escombro en compañía de gasterópodos grandes. Manual; 2002; Sormani, H. C. G. col. (IEXA). $1 \lesssim$ Xalapa Enríquez, Infonavit Sumidero. Zona Urbana. En jardín sobre muro. Manual; enero 2008;

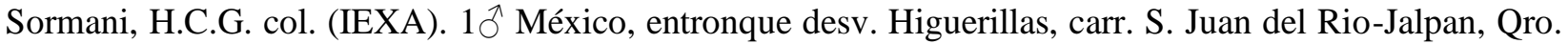
(VIII-B), en detritos de Atta mexicana, matorral de Larrea, $1420 \mathrm{~m} \mathrm{snm;} \mathrm{31/V/1983;} \mathrm{P.} \mathrm{Rojas} \mathrm{col.} \mathrm{(CBS)}$ [det. F. W. Fisk, 1983]. 2 ふ̋ México, entronque desv. Higuerillas, carr. S. Juan del Rio-Jalpan, Qro. (VIIIA), en detritos de Atta mexicana, matorral de Larrea, $1420 \mathrm{~m} \mathrm{snm;} \mathrm{11/IV/1982;} \mathrm{P.} \mathrm{Rojas} \mathrm{col.} \mathrm{(CBS)} \mathrm{[det.}$ F.W. Fisk, 1983]. Compsodes schwarzi (Caudell, 1903) 3 กิ ô México, Jalisco, Est. Biol. Chamela, Cuenca 1, trampa maisle; 18-23/VIII/1992; A. Rodríguez col. (LESM).

\section{DISCUSIÓN}

La hembra de Eremoblatta atticola sp. n. presenta mayor adaptación a una vida mirmecófilas, con: 1) talla reducida (9 mm, Figs. 15, 16); 2) cuerpo cubierto por cerdas; 3) reducción de apéndices (Figs. 18-20); 4) patas fosoriales (Figs. 18-20); 5) cercis cortos y retraído (Figs. 21, 22) (Vršanský et al., 2019 [2018]); 6) ausencia de tegminas y alas (reducción de alas sensu Bell et al., 2007) (Figs. 15-16); 7) ojos reducidos (Fig. 17). Mientras que el macho presenta mayor adaptación a vida libre (Figs. 1-8), a pesar de poseer gran cantidad de cerdas, sus patas son muy largas y con los tarsómeros notablemente alargados (Figs. 4-6), ojos desarrollados (Fig. 3), antenas largas y alas macropteras (Figs. 7, 8).

Las especies de cucarachas que viven con hormigas del género Atta se dispersan de colonia en colonia siguiendo las columnas de forrajeo de las hormigas, o trepando a las reinas que salen al vuelo nupcial (Moser, 1964; 1967). Sin embargo, hasta donde sabemos, se desconoce de qué forma llegan a los detritos, aunque podemos suponer que son atraídas por el olor característico que emana de ellos (Rojas, 1989).

El hallazgo de adultos de ambos sexos y de ninfas de E. atticola sp. n. dentro de los montículos de detritos, así como la observación de una pareja en cópula, nos indican que probablemente estos insectos pasan todo su ciclo de vida en este ambiente. Se sabe que los desechos orgánicos de los nidos de las hormigas del género Atta inciden en una mayor abundancia y riqueza de la biota del suelo (Rojas, 2003; Farji-Brener \& Tadey, 2009), lo que es particularmente importante en zonas secas, en donde los recursos son escasos (Bestelmeyer \& Wiens, 2003). Eremoblatta atticola sp. n. es un claro ejemplo de una especie favorecida por las acumulaciones de detritos de Atta mexicana en una zona árida de México, aseveración que apoyamos con el hecho de que no se le ha encontrado fuera de este ambiente.

AgRAdeCiMIEnTOS. Sinceramente agradecemos al Dr. Alejandro Zaldívar R. y a la M. en C. Ma. Cristina Mayorga M. (CNIN-IBUNAM, UNAM, CDMX, México); Biól. Delfino Hernández (IEXA-INECOL, Veracruz, México); Biól. Antonio Ángeles (Departamento Biología de Suelos del INECOL); Dr. José G. Palacios Vargas y Dra. Blanca E. Mejía Recamier (Laboratorio de Ecología y Sistemática de 
Microartrópodos, Fac. Ciencias, UNAM. México), por las facilidades otorgadas para la revisión de material incluido en este estudio. A Entomological Research por financiar parcialmente este proyecto.

\section{LITERATURA CITADA}

Ball, E. D., Tinkham, E. R., Flock, R., Vorhies, C. T. (1942) The grasshoppers and other Orthoptera of Arizona. University of Arizona College Agricultural Experiment Station Technical Bulletin, 93, 257-373.

Beccaloni, G. W. (2014) Blattodea Species File Online. Version 5.0/5.0.World Wide Web electronic publication. Disponible en: http://Cockroach.SpeciesFile.org (ultimo acceso 2 de agosto 2019).

Beccaloni, G. W., Eggleton, P. (2011) Order Blattodea Brunner von Wattenwyl, 1882. Pp: 199-200. In: Z. Q. Zhang (Ed.). Animal biodiversity: An outline of higher-level classification and survey of taxonomic richness. Zootaxa, 3148. https://doi.org/10.11646/zootaxa.3148.1.37

Beccaloni, G. W., Eggleton, P. (2013) Order Blattodea Brunner von Wattenwyl, 1882. Pp: 46-48. In: Z. Q. Zhang (Eds.). Animal Biodiversity: An Outline of Higher-level Classification and Survey of Taxonomic Richness (Addenda 2013). Zootaxa, 3703. https://doi.org/10.11646/zootaxa.3148.1.37

Beebe, W. (1921) Edge of the jungle. Garden City, N.Y., Garden City Publishing, 312 pp. https://doi.org/10.5962/bhl.title.55090

Bell, W. J., Roth, L. M., Nalepa, C. A. (2007) Cockroaches: ecology, behavior, and natural history. John Hopkins University Press, Baltimore, 237 pp.

Bestelmeyer, B. T., Wiens, J. (2003) Scavenging ant foraging behavior and variation in the scale of nutrient redistribution among semi-arid grasslands. Journal of Arid Environments, 53, 373-86. https://doi.org/10.1006/jare.2002.1044

Bolívar, I. (1905) Les blattes myrmécophiles. Mitteilungen der Schweizerischen Entomologischen Gesellschaft=Bulletin de la Société entomologique suisse, 11, 134-141. https://doi.org/10.5962/bhl.part.2751

Brossut, R. (1976) Étude morphologique de la Blatte myrmécophile Attaphila fungicola Wheeler. Insectes Sociaux, 23 (2), 167-174. https://doi.org/10.1007/bf02223849

Bruch, C. (1916) Contribución al estudio de las hormigas de la provincia de San Luis. Revista del Museo de La Plata, 23, 291-357. https://doi.org/10.24215/25456377e040

Bruijning, C. F. A. (1959) The Blattidae of Surinam. Studies on the Fauna of Suriname and other Guyanas, 2, 1-103.

Caudell, A. N. (1913) Notes on Nearctic Orthopterous Insects. I Nonsaltatorial forms. Proceedings of the United States National Museum, 44, 595-614. https://doi.org/10.5479/si.00963801.44-1970.595

Caudell, A. N. (1923) Phorticolea boliviae, a new myrmecophilous cockroach from South America. Psyche, 30, 28-30. https://doi.org/10.1155/1923/19248

Chopard, L. (1924a) Description d'un blattide myrmecophile nouveau (Orth.). Bulletin de la Société entomologique de France, 29 (11-12), 131-132. https://doi.org/10.5962/bhl.part.16275

Chopard, L. (1924b) Rectification a propos d'un blattide myrmecophile (Orth.). Bulletin de la Société entomologique de France, 29 (17), 186.

Crespo, F. A., Di Iorio, O., Valverde, A. C. (2015) Contributions to the knowledge of Hypercompsa, new register from Argentina (Blattaria: Corydiidae). Revista de la Sociedad Entomológica Argentina, 74 (3-4), 203-207. 
Deans, A. R., Roth, L.M. (2003) Nyctibora acaciana (Blattellidae: Nyctiborinae), a new species of cockroach from Central America that oviposits on ant-acacias. Transactions of the American Entomological Society, 129, 267-283.

Deyrup, M. A., Fisk, F. W. (1984) A myrmecophilous cockroach new to the United States (Blattaria: Polyphagidae). Entomological News, 95, 183-185.

Estrada-Álvarez, J. C. (2013) Primera lista de las Cucarachas (Insecta: Dictyoptera: Blattaria) de México. Boletín de la Sociedad Entomológica Aragonesa, 53, 267-284.

Estrada-Álvarez, J. C., Guadarrama, C. (2013) Una especie nueva de Myrmecoblatta Mann, 1914 (Blattaria: Polyphagidae: Latindinae) de México. Boletín de la Sociedad Entomológica Aragonesa, 52, 93-95.

Farji-Brener, A. G., Tadey, M. (2009) Contributions of leaf-cutting ants to soil fertility: causes and consequences. Pp: 81-91. In: P. Derek, C. Lucero, J. Boggs (Eds.). Soil Fertility. Nova Science Publishers, New York.

Fisk, F. W., Vargas, M., Fallas, F. (1976) Notes on Myrmecoblatta Weeleri from Costa Rica (Blattaria: Polyphagidae). Proceedings of the Entomological Society of Washington, 78, 317-322.

Grandcolas, P. (1994) Phylogenetic systematics of the subfamily Polyphaginae, with the assignment of Cryptocercus Scudder, 1862 to this taxon (Blattaria, Blaberoidea, Polyphagidae). Systematic Entomology, 19, 145-158. https://doi.org/10.1111/j.1365-3113.1994.tb00584.x

Gurney, A. B. (1937) Studies in certain genera of American Blattidae (Orthoptera). Proceedings of the Entomological Society of Washington, 37, 101-112.

Gurney, A. B., Roth, L. M. (1972) A generic review of the cockroaches of the subfamily Panchlorinae (Dyctioptera, Blattaria, Blaberidae). Annals of the Entomological Society of America, 65 (3), 521532. https://doi.org/10.1093/aesa/65.3.521

Hancock, G. L. R. (1926) A winter entomological visit to Central Brazil. Entomologist, 59 (49-52), 131137.

Hebard, M. (1917) The Blattidae of North America North of the Mexican boundary. Memoirs American Entomological Society, 2, 284 pp.

Hendrichs. J., Reyes-Castillo, P. (1963) Asociación entre coleópteros de la familia Passalidae y hormigas. Ciencia (México), 22 (4), 101-104.

Hopkins, H. (2014) A revision of the genus Arenivaga (Rehn) (Blattodea, Corydiidae), with descriptions of new species and key to the males of the genus. ZooKeys, 384, 1-256. https://doi.org/10.3897/zookeys.384.6197

INEGI (Instituto Nacional de Estadística y Geografía) (2020) Mapa Digital de México V6.3.0. Disponible en: http://gaia.inegi.org.mx/mdm6

Jones, C. G., Lawton, J. H., Shachak, M. (1994) Organisms as ecosystem engineers. Oikos, 69, 373-386. https://doi.org/10.2307/3545850

Maes, J-M. (1992) Catálogo de los Blattodea (Dictyoptera) de Nicaragua. I Familias Polyfagidae y Blaberidae. Revista Nicaragüense de Entomología, 19, 21-28.

Mann, W. (1914) Some Myrmecophilous Insects from México. Psyche, 21, 171-183. https://doi.org/10.1155/1914/69251

Morrone, J. J. (2001) Biogeografía de América Latina y el Caribe. M\&T-Manuales \& Tesis SEA, 3. Zaragoza, 148 pp.

Morrone, J. J., Márquez, J. (2008) Biodiversity of Mexican terrestrial arthropods (Arachnida and Hexapoda): a biogeographical puzzle. Acta Zoológica Mexicana (nueva serie), 24 (1), 15-41. https://doi.org/10.21829/azm.2008.241613

Moser J. C. (1964) Inquiline roach responds to trail-marking substance of leaf cutting ants. Science, 1048, 143.

https://doi.org/10.1126/science.143.3610.1048 
Moser J. C. (1967) Mating activities of Atta texana (Hymenoptera, Formicidae). Insectes Sociaux, 4, 295312. https://doi.org/10.1007/bf02252831

Nehring, V., Dani, F. R., Calamai, L., Turillazzi, S., Bohn, H., Klass, K. D., d'Ettorre, P. (2016) Chemical disguise of myrmecophilous cockroaches and its implications for understanding nestmate recognition mechanisms in leaf-cutting ants. BMC Ecology, 35, 1-11. https://doi.org/10.1186/s12898-016-0089-5

Phillips, Z. I., Zhang, M. M., Mueller, U. G. (2017) Dispersal of Attaphila fungicola, a symbiotic cockroach of leaf-cutter ants. Insectes Sociaux, 64 (2), 277-284. https://doi.org/10.1007/s00040-016-0535-6

Princis, K. (1963) Blattariae: Suborder [sic] Polyphagoidea: Fam.: Homoeogamiidae, Euthyrrhaphidae, Latindiidae, Anacompsidae, Atticolidae, Attaphilidae. Suborder Blaberoidea: Fam. Blaberidae. Pp: 76-172. In: M. Beier (Ed.). Orthopterorum Catalogus. Pars 4. W. Junk's-Gravenhage. https://doi.org/10.1002/mmnz.4830400108

Rehn, J. (1903) A Revision of the Orthopterous Genus Homoeogamia. Proceedings of the Academy of Natural Sciences of Philadelphia, 55, 177-192.

Rehn, J. (1906) The Orthoptera of the Bahamas. Bulletin of the American Museum of Natural History, 22, $107-118$.

Renh, J., Hebard, M. (1927) The Orthoptera of the West Indies. Number I. Blattidae. Bulletin of the American Museum of Natural History, 54, 1-320.

Rodríguez, J. G., Montoya-Lerma, J., Calle, Z. D. (2013) Primer registro de Attaphila fungicola (Blattaria: Polyphagidae) en nidos de Atta cephalotes (Hymenoptera: Myrmicinae) en Colombia. Boletin Cientifico Centro De Museos De Historia Natural, 17 (1), 219-225. https://doi.org/10.17151/bccm.2015.19.1.15

Rojas, P. (1989) Entomofauna asociada a los detritos de Atta mexicana (F. Smith) (Himenoptera: Formicidae) en una zona árida del centro de México. Acta Zoológica Mexicana (nueva serie), 33, 3-51. https://doi.org/10.21829/azm.2015.312990

Rojas, P. (2003) El papel de las hormigas (Hymenoptera: Formicidae) en la dinámica edáfica. Pp: 197-216. In: Álvarez-Sánchez, Naranjo García (Eds.). Ecología del Suelo en la Selva Tropical Húmeda de México. Instituto de Ecología A.C./Instituto de Biología/ Facultad de Ciencias, UNAM. Ciudad de México, México. https://doi.org/10.3926/oms.353

Roth, L. M. (1995) Pseudoanaplectinia yumotoi, A New Ovoviviparous Myrmecophilous Cockroach Genus and Species from Sarawak (Blattaria: Blattellidae; Blattellinae). Psyche, 102 (1-2), 79-87. http://dx.doi.org/10.1155//92482

Roth, L. M. (2003) Systematics and Phylogeny of Cockroaches (Dictyoptera: Blattaria). Oriental Insects, $37,1-186$. https://doi.org/10.1080/00305316.2003.10417344

Roth, L. M., Willis, E. R. (1960) The biotic associations of cockroaches. Smithsonian Miscellaneous Collections, 141, 1-470.

Vršanský, P., Śmídová, L., Sendi, H., Barna, P., Müller, P., Ellenberger, S., Wu, H., Ren, X., Lei, X., Azar, D., Šurka, J., Su, T., Deng, W., Shen, X., Lv, J., Bao, T., Bechly, G. (2019) [en linea 2018]. Parasitic cockroaches indicate complex states of earliest proved ants. Biologia, 74 (1), 65-89. https://doi.org/10.2478/s11756-018-0146-y

Walter, E. V., Seaton, L., Mathewson, A. A. (1938) The Texas leaf-cutting ant and its control. Circular U.S.D.A., 494, 1-19.

Weber, N. A. (1972) Gardening ants: the attines. Memoirs of the American Philosophical Society, 92, 1146.

https://doi.org/10.1126/science.178.4063.856 
Estrada-Álvarez \& Rojas: Nueva cucaracha mirmecófila

Wheeler, W. M. (1900) A New Myrmecophile from the Mushroom Gardens of the Texan Leaf-cutting Ant. The American Naturalist, 34 (407), 851-862. https://doi.org/10.1086/277806

Wheeler, W. M. (1907) The fungus-growing ants of North America. Bulletin of the American Museum of Natural History, 23 (XXXI), 669-807.

Wheeler, W. M. (1910) Ants: their structure, development and behavior. University Press, New York. Columbia, 696 pp.

Wheeler, W. M. (1928) The social insects. Their origin and evolution. New York, 378 pp. 